\title{
High-accuracy filtered neutron beam and high-energy transmission measurements at the Gaerttner Laboratory
}

\author{
Y. Danon ${ }^{1, a}$, R.C. Block ${ }^{1}$, M. Rapp ${ }^{1}$, F. Saglime ${ }^{1}$, D.P. Barry ${ }^{2}$, N.J. Drindak ${ }^{2}$, J. Hoole ${ }^{2}$, and G. Leinweber ${ }^{3}$ \\ 1 Gaerttner Laboratory, Rensselaer Polytechnic Institute, Troy, NY 12180, USA \\ 2 Lockheed Martin Corporation, Schenectady, NY 12301-1072, USA
}

Abstract. Recently a method for high accuracy total cross section measurement in the energy range of $24 \mathrm{keV}$ to $940 \mathrm{keV}$ using an iron filtered beam was developed at RPI. Measurements the total cross section of carbon and beryllium are discussed. A new neutron detection system was developed at RPI and the first measurement with this system is reported here.

\section{Filtered beam measurements}

The idea of a filtered beam measurement was previously explored at RPI for transmission measurements of deuterium [1]. Recently a ${ }^{238} \mathrm{U}$ filtered beam was also used [2] and further explored in ref. [3]. The iron filtered beam utilized a $30 \mathrm{~cm}$ thick iron piece placed in a collimated neutron beam between the neutron source and the sample being measured. The experiment was done using the conventional time-offlight (TOF) method with the detector placed at a flight path distance of $25.55 \mathrm{~m}$.

Most of the neutrons that interact with the thick iron filter are removed from the beam. At neutron energies corresponding to resonance potential interference where the total cross section exhibits a minimum, the filter is nearly transparent to neutrons. The resulting filtered beam has about 19 peaks of intense neutron flux; in some the transmission through the filter is $60 \%$. The main advantage of the filtered beam method is the removal the time dependent neutron and gamma background which imposes limitations on the accuracy of TOF measurements. In a filtered beam experiment the typical signal to noise ratio varies between 500-5000 depending on the selected peak. The accuracy obtained for graphite and beryllium total cross sections measured using this method is about $1 \%$. Better accuracy can be obtained with more careful attention to details such as dead time correction and beam stability normalization.

We tested the filtered beam method with a sample of graphite (carbon) for which the total cross section is well known. The method was then used on beryllium. Open beam data is shown in figure 1 . It is interesting to note that the counts between the resonances are mostly dominated by resolution broadening and not off energy background neutrons or gammas. The background is mainly room background and neutrons that pass around the filter and can be measured at long time of flight. Transmission is calculated by integrating the peak in the open and sample data, correcting each for the background, and taking the ratio of the background corrected sample to the open count rates. The transmission is converted to cross section by the simple relation $\sigma=-\ln (T) / N$ where

${ }^{a}$ Presenting author, e-mail: danony@rpi .edu

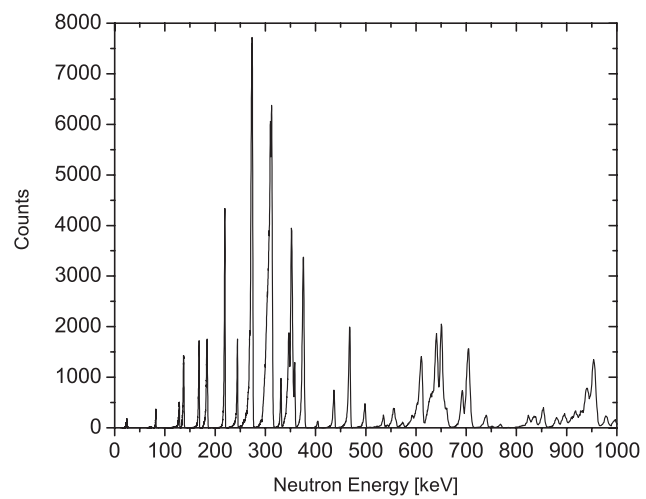

Fig. 1. TOF spectrum of a $30 \mathrm{~cm}$ thick Fe filtered open beam (the TOF was converted to neutron energy).

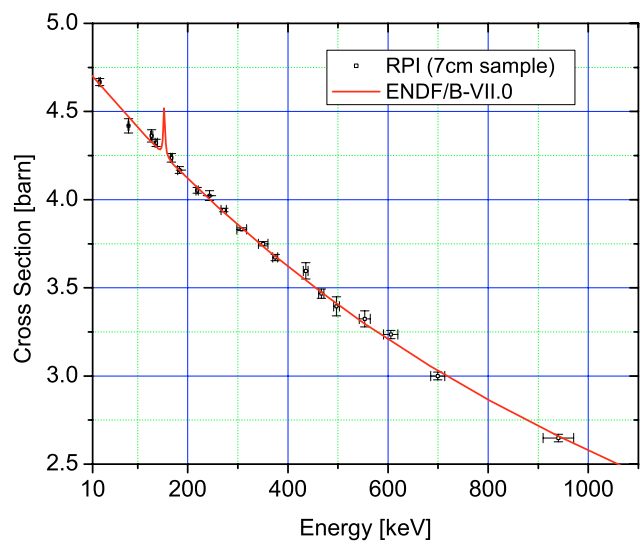

Fig. 2. The total cross section measured using a $7 \mathrm{~cm}$ thick graphite sample compared with the ENDF/B-VII.0 evaluation for carbon. The energy error bar represents the width of the peak (in fig. 1) that was summed.

$T$ is the transmission and $N$ [atom/barn]is the atom density of the sample. The total cross section of a $7 \mathrm{~cm}$ thick graphite sample is shown in figure 2 together with the ENDF/B-VII.0 [4] evaluation for ${ }^{12} \mathrm{C}$. All the points with statistical error of $1 \%$ or less are within $1 \%$ of the evaluation. The data is in 


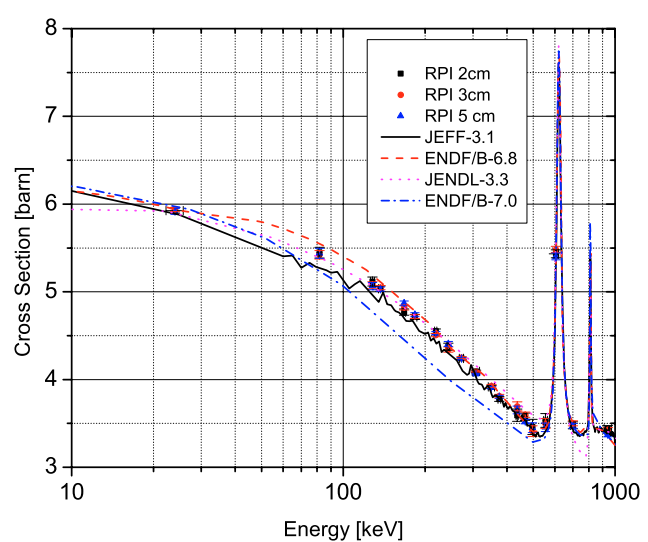

Fig. 3. The measured total cross section of beryllium plotted with several evaluations.

excellent agreement with the ENDF and can be interpreted as a verification of our method or the quality of the ENDF data for carbon. The sample used was reactor grade graphite with density of $1.686 \mathrm{~g} / \mathrm{cm}^{3}$ and was baked for 24 hours to remove moisture from the sample.

The measured total cross section for $2 \mathrm{~cm}$ and $3 \mathrm{~cm}$ thick samples of beryllium ( $99.9 \%$ pure) are shown in figure 3 . The data deviates from all current evaluations in different energy ranges. The JENDL-3.3 seems to have the best fit but is slightly higher than the data in the energy range of $300-500 \mathrm{keV}$. The new ENDF/B-VII.0 evaluation is the most discrepant.

\section{High energy transmission measurements}

A new detector was recently developed at RPI [5]. The detector is a modular EJ301 liquid scintillator. Each of the six modules has dimensions of $35.5 \mathrm{~cm} \times 18 \mathrm{~cm}$ by $13.8 \mathrm{~cm}$ thick with two $12.7 \mathrm{~cm}$ diameter photomultipliers (PMTs). The PMTs are connected directly to an ORTEC CF8000 discriminator. The array is operated in OR geometry. The data are collected using a FAST ComTec P7889 TOF clock. The detector is located at a flight path of about $100 \mathrm{~m}$ and the RPI LINAC delivered electron pulse width of $6 \mathrm{~ns}$. The neutron target is water cooled tantalum placed on the neutron beam axis. In order to reduce the gamma background a $7.62 \mathrm{~cm}$ thick depleted uranium filter was used. To benchmark the system in the energy range from $0.4 \mathrm{MeV}$ to $20 \mathrm{MeV}$ the well known total cross section of carbon was measured. Two reactor grade graphite samples with thickness of $7 \mathrm{~cm}$ and $13 \mathrm{~cm}$ were measured. The transmission data are shown in figure 4 compared to ENDF/B-VII.0 broadened to $300 \mathrm{~K}$. The data are in good agreement with the evaluation; however, some sharp resonances are not well resolved. This resolution issue was expected for this measurements technique, but will not affect the measurement accuracy for materials in energy

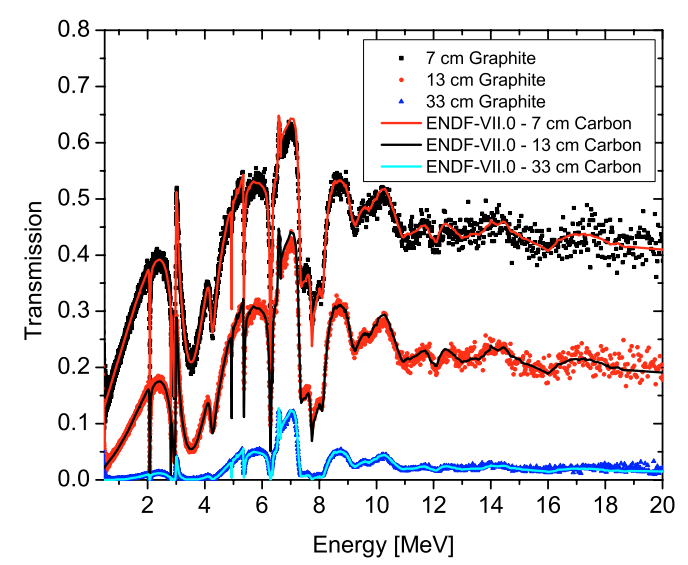

Fig. 4. Transmission measurements of two graphite samples.

ranges with smooth cross section or with broad resonance structure.

\section{Conclusions}

New experiments at the RPI LINAC utilized an iron filtered beam to measure the total cross section of graphite and beryllium samples with an accuracy of $1 \%$. Such a measurement provided several accurate points in the energy range from $24 \mathrm{keV}$ to $940 \mathrm{keV}$. The method was benchmarked on graphite and was applied to beryllium. The carbon data are in excellent agreement with ENDF/B-VII.0 but the beryllium data differs from all current cross section evaluations indicating a need for improvement in those evaluations.

A new large area modular transmission detector was installed at the $100 \mathrm{~m}$ flight path of the Gaerttner LINAC Laboratory at RPI. This detector enables neutron transmission measurements in the energy range of $0.4 \mathrm{MeV}$ to $20 \mathrm{MeV}$. Results with several graphite samples show good agreement with the ENDF/B-VII.0 evaluation. The energy resolution of the system is sufficient to measure materials with smooth cross sections or with broad resonance structure but not enough to resolve the sharp resonances in carbon.

\section{References}

1. P. Stoler, N.N. Kaushal, F. Green, Phys. Rev. C 8, 1539 (1973).

2. R. Moreh, R.C. Block, Y. Danon, M. Neuman, Phys. Rev. Lett. 96, 055302 (2006).

3. R. Moreh, R.C. Block, Y. Danon, NIM A 562, 401 (2006).

4. M. Chadwick, P. Obložinský, M. Herman et al., Nucl. Data Sheets 107, 2931 (2006), http://www.sciencedirect.com/science/journal/00903752.

5. M. Rapp, D. Yaron, B. Robert, The American Nuclear Society's 14th Biennial Topical Meeting of the Radiation Protection and Shielding Division, Carlsbad New Mexico, USA, April 3-6 2006, No. 93 (2006), p. 512. 